$\begin{array}{ll} & \text { Etnográfica } \\ \text { etnográfica } & \text { Revista do Centro em Rede de Investigação em }\end{array}$

Antropologia

vol. $13(1) \mid 2009$

Vol. $13(1)$

\title{
Le peloton cycliste : de la métaphore sociale au bouillon de culture
}

The bunch of cyclists: from social metaphor to culture medium

\section{Xavier Garnotel}

\section{(2) OpenEdition}

\section{Journals}

Édition électronique

URL : https://journals.openedition.org/etnografica/1301

DOI : 10.4000/etnografica.1301

ISSN : 2182-2891

\section{Éditeur}

Centro em Rede de Investigação em Antropologia

\section{Édition imprimée}

Date de publication : 2 mai 2009

Pagination : 17-30

ISSN : 0873-6561

\section{Référence électronique}

Xavier Garnotel, «Le peloton cycliste : de la métaphore sociale au bouillon de culture », Etnográfica [En ligne], vol. 13 (1) | 2009, mis en ligne le 16 mars 2012, consulté le 07 décembre 2022. URL : http:// journals.openedition.org/etnografica/1301 ; DOI : https://doi.org/10.4000/etnografica.1301

\section{(c) (†) \&)}

Creative Commons - Attribution - Pas d'Utilisation Commerciale 4.0 International - CC BY-NC 4.0 https://creativecommons.org/licenses/by-nc/4.0/ 


\section{Le peloton cycliste : de la métaphore sociale au bouillon de culture ${ }^{1}$}

\section{Xavier Garnotel}

Cet article présente dans un premier temps l'enchâssement du sport cycliste dans les structures techniques et sociales des sociétés occidentales. Des notes ethnographiques décrivent ensuite la vie du peloton et les pratiques des coureurs. Ces descriptions illustrent des traits de culture inédits et parfois même en décalage avec les textes réglementaires. L'analyse porte alors sur l'adaptation des pratiquants vis-à-vis des propriétés de la pratique et de la construction de codes sociaux et d'une culture technique spécifique.

MOTS-CLÉS: ethnologie, culture, sport, peloton, cyclisme, règlement.

\section{LES VALEURS FONDATRICES DU CYCLISME “ TRADITIONNEL ”}

Le sport cycliste a été structuré par les valeurs des sociétés occidentales. Tout comme les autres grands sports-spectacles, la course en peloton représente une métaphore des modèles organisationnels et des valeurs du monde contemporain où se structurent : " trois principes opposés dont la conjonction forme [...] le canevas d'une vision contradictoire mais cohérente du monde" : le mérite individuel (idéal mérito-démocratique), l'organisation et la solidarité collective dans la tâche (modèle industriel), et la place du hasard, de l'aléa et même de la duperie. "En un même mouvement, le match de football, où se conjuguent le mérite individuel et collectif, la chance, la friponnerie, la décision opportune, exhibe ainsi les facteurs déterminants de la réussite dans le monde contemporain et, à travers ses propriétés incertaines, une palette d'interprétations acceptables de l'échec. Il peut nourrir et réunir par là même une culture positive du succès prométhéen et une philosophie compréhensive de l'infortune résignée " (Bromberger 1995a: 203).

l Je tiens à remercier Jean-Yves Durand et Christian Bromberger pour leur lecture et leurs critiques. 
La course cycliste constitue également une métaphore du modèle méritocratique par son alternative essentielle : sortir du peloton ou lutter pour y rester. Elle représente aussi des valeurs industrielles modernes : surmonter les difficultés, encaisser des charges de travail dans la douleur et l'abnégation, souffrir en groupe, se glisser dans les bons coups, ruser, faire des alliances parfois fourbes, ne pas dévoiler son jeu, surprendre et affirmer son autorité ; traverser des moments d'euphorie et de prestige : s'échapper, se détacher et avoir les honneurs, la reconnaissance ; être dépassé, doublé, lâché, se retrouver seul au plus bas...

La pratique cycliste s'intègre également dans le mobilier technique des sociétés industrielles. L'évolution des systèmes techniques et des matériaux de la bicyclette s'enchâsse parfaitement dans les modèles technologiques et économiques européens du $19^{\text {ème }}$ et du $20^{\text {ème }}$ siècles. De l'usage ludique et aristocratique des premiers vélocipèdes au cours du second empire, à la " petite reine " symbole de la locomotion populaire et ouvrière jusqu'aux vélos hi-tech agrégeant des matériaux composites issus de l'aéronautique, cet engin cristallise les techniques industrielles de nos sociétés.

Jusqu'aux premières études ethnologiques réalisées sur la course à pied (Segalen 1994), le football (Bromberger 1995a), le rugby (Darbon 2002; Saouter 1995), ou encore la boxe (Wacquant 2002), le cyclisme, comme les autres pratiques sportives, a été uniquement abordé sous des perspectives historiques, technologiques et sociologiques. Elles étaient alors entrevues comme des synthèses techniques, institutionnelles et socio-économiques des sociétés dans lesquelles elles s'inséraient, une forme de miroir social. Mon expérience de pratiquant a alimenté des interrogations et une insatisfaction théorique. Sans remettre en question l'apport indispensable de ces perspectives sociohistoriques, je ne reconnaissais pas ce que j'avais pu voir ou entendre dans le peloton à travers ces analyses. Pour envisager une pratique à travers cet angle, seule une méthodologie compréhensive paraissait pertinente. J'ai donc réalisé une enquête de terrain de deux ans (saisons 2002 et 2003) au sein du peloton amateur et professionnel associant observation participante classique et participation observante. L'essentiel des notes a été réalisé sur des courses de l'élite amateur (l'équivalent de la deuxième division pour les sports collectifs), rassemblant des équipes françaises, espagnoles, italiennes et africaines (lors du Tour de Tunisie). Les matériaux ethnographiques ont été rédigés sous la forme d'une monographie (Garnotel 2009). En voici quelques extraits qui seront à la base des interprétations formulées dans cet article. 


\section{NOTES ETHNOGRAPHIQUES ET PROBLÉMATIQUE}

\section{Février 2003 : De "l'échappée au grupetto"}

Le dimanche matin, nous nous préparons de nouveau pour la dernière de ces " courses au soleil " : " la ronde du Canigou " entre Lloret del Mar et Perpignan. Comme d'habitude, ça part sur les " chapeaux de roue ". Un élément va changer la donne de cette course : le vent est de face. Cette configuration désavantage les attaquants et favorise les coureurs " justes " qui peuvent rester "bien au chaud au milieu du paquet" alors que les "costauds se cassent les dents devant ". Les attaques se succèdent et une échappée de 20 coureurs se dessine. Alors que je pensais que la course était finie (pas au niveau des kilomètres mais de son issue), j'aperçois quatre équipes (Wasquehal, Etupes, Châteauroux et une équipe espagnole) remonter le peloton et s'organiser devant. Le peloton revient sur l'échappée à 20 kilomètres du col du Perthus. Je pars dans une échappée d'une quinzaine de coureurs. Toutes les grosses équipes sont représentées mais l'entente n'est pas bonne. "Ça se pose ", ça attaque dans tous les sens. Nous arrivons dans le Perthus, je suis "dans la roue" du champion d'Italie. Les spectateurs crient et tapent des mains, je ne sens plus la douleur, ou plutôt je la sens et l'apprécie, la dépasse, “j'me fais péter les varices". Un parfum, une aura indescriptible se dégagent de ce moment. Le groupe " explose ". Quatre coureurs se détachent. Je suis dans le groupe de contre avec quatre autres coureurs. Malgré cela, nous nous faisons " absorber " dans la descente par le peloton tout comme le groupe de tête. Le vent de face défavorise les attaques. Dans la descente, le capitaine de route, Sébastien, monte à ma hauteur pour me dire que nous allons " choper le vent de côté les 30 derniers kilomètres à partir du Boulou ”. A l'entrée du rond point du Boulou, la tension est insupportable, "ça frotte" (coureurs qui se touchent et se poussent avec les coudes pour se placer) et devient très dangereux. Les voitures garées sur le côté sont frôlées de quelques centimètres. Tout d'un coup " on attrape le vent de côté ". A partir de là, " c'est le chantier". On roule " à bloc " et le compteur est à $25 \mathrm{~km} / \mathrm{h}$. Le vent, quant à lui, doit souffler à plus de $70 \mathrm{~km} / \mathrm{h}$. En plus de cela, je suis mal placé, je " bouche des trous " laissés par des coureurs " qui sautent ", " qui pètent ", " qui explosent " (se font lâcher). Je vois un coureur avec de la bave et de la morve partout sur le visage. La douleur est insoutenable, je me retiens de crier de douleur alors qu'enfin j'arrive à m'abriter. Les coureurs ont constitué un " éventail ". Il y en a deux autres devant nous. Derrière "y'en a partout, ça a explosé de tous les côtés". Les 15 derniers kilomètres sont pénibles. L'ambiance du groupe s'humanise un peu. Les coureurs battus de ce groupe échangent quelques brefs commentaires. Nous arrivons pour la trentième place. Personne ne fait le sprint. Les quelques mètres qui séparaient les "éventails" à leur origine se sont transformés en minutes. Les autres membres de l'équipe arrivent dans le "grupetto". 


\section{Août 2002 : Entrer dans la mafia!}

Voici maintenant le récit d'une course élite-nationale sur un critérium champêtre à la fin de l'été de la saison précédente (2002). Une " mafia " composée d'une dizaine de coureurs sur les 80 engagés s'était organisée. Autrement dit, des coureurs de clubs différents réalisent une entente pour faire gagner un coureur qui donne de l'argent. Après dix kilomètres, le peloton explose et je rejoins le groupe de tête, nous formons une échappée de cinq coureurs. Alors que je récupère de cet effort en restant quelques instants " dans les roues ", j'entends une ferme altercation entre mon coéquipier Sébastien et le chef de la mafia. Sébastien ne veut pas entrer dans cette entente, l'autre coureur lui dit qu'il ne joue pas le jeu et qu'il ne comprend rien au vélo. Le coureur qui souhaite gagner devant ses proches et son village natal explique : " de toutes façons, les plus forts sont là, si tu refuses, on se reformera dans 20 bornes et le problème sera le même ". Pour ma part, je suis “ à fond dans les roues " et je n'ai pas mon mot à dire. Sébastien finit par accepter d'entrer dans la " cabane " (synonyme de "mafia "). Le cinquième coureur refuse d'entrer dans cette entente. Il ne collabore pas aux relais et dispute les primes (argent distribué lors de la course provenant de dons de spectateurs). A mi-course, nous mettons un tour au peloton. Des coureurs doublés s'intercalent dans l'échappée et disputent certaines primes, c'est la foire d'empoigne. Le chef de la " mafia " trouve ces comportements inacceptables. A quelques kilomètres de la fin, chacun réalise de fausses attaques pour le spectacle et le coureur qui donne l'argent remporte sa course. Après avoir récupéré nos prix de courses au bar du village, nous nous retrouvons plus loin, et le chef de la mafia fait les comptes sur le capot de sa voiture. Il émet des commentaires sur le comportement du cinquième coureur : " c'est inacceptable des mecs pareils, ils ont aucune morale, il roule pas avec nous et il nous fait les primes, heureusement que j'étais pas chargé [dopé] aujourd'hui sinon je l'aurais mis dans le fossé ". Il réalise le partage des 150 euros versés par le gagnant. Avec les prix de course, ce dernier a équilibré ses gains et dépenses, les autres bénéficient des sommes officielles et officieuses.

Ces quelques récits de course entrent en contradiction avec les discours officiels des représentants institutionnels et sont parfois en rupture avec les règlements écrits. Ils paraissent enfin très éloignés des valeurs fondatrices qui accompagnent la définition des sports basés sur les principes moraux d'égalité des chances, de mérite et d'une certaine forme de désintérêt du gain matériel pour la logique amateuriste coubertinienne. Cette éthique reposait sur la volonté aristocratique de maintenir une distinction sociale en se faisant garante d'une mentalité sportive noble et désintéressée. Mais le sport cycliste s'est démarqué dès la fin du $19^{\text {ème }}$ siècle par l'émergence d'une forte dynamique professionnelle sous l'impulsion des célèbres marques de cycles qui parrainaient les grandes épreuves à des fins de notoriété. Si bien que l'amateurisme a rapidement été substitué par une culture populaire plus pragmatique. 
En effet, en favorisant des systèmes de récompenses généreux, l'Union Vélocipédique de France créée en 1890, a incité les coureurs des classes populaires à participer aux épreuves et à remporter ces gains. Par exemple, le Grand Prix de l'Union Vélocipédique de France offrait 10000 francs au vainqueur alors qu'à titre indicatif, le salaire annuel d'un instituteur était environ de 1000 francs (Lagrue 2004). Ce système de récompense et cette appropriation populaire de la pratique ne sont sans doute pas étrangers à la régulation spontanée du cyclisme sur route. Les gains financiers font partie intégrante de la pratique cycliste dès les jeunes catégories. L'intérêt pour l'argent se transmet à travers l'oralité du peloton et avec les pratiques spécifiques des courses sur route, notamment lors des critériums où sont distribuées des primes indépendantes du résultat final.

Pourtant, ceci n'explique ni les fondements de la culture technique du peloton ni le mécanisme de construction de codes sociaux spécifiques à ce sport. L'hypothèse de cet article est que les propriétés physiques, techniques et réglementaires de la pratique cycliste sont à l'origine d'adaptations pragmatiques des pratiquants qui ont construit des traits de culture propres au peloton cycliste.

\section{LADAPTATION TECHNIQUE AUX EXIGENCES PHYSIQUES ET PHYSIOLOGIQUES}

Le premier extrait des notes ethnographiques mentionne l'existence " d'éventails". Il s'agit d'une des organisations techniques collectives construites pragmatiquement pour s'adapter aux caractéristiques physiques de l'effort du cyclisme sur route. En temps normal, les coureurs se placent les uns derrière les autres pour se protéger de la résistance de l'air au mouvement :

\section{H}

Mais lorsque le vent est latéral (dans cet exemple, le vent viendrait du haut) ils se décalent, cela fait un "éventail" :

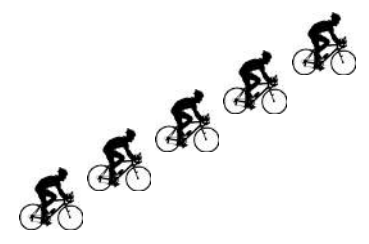


Pour organiser les relais, l'éventail possède deux files si le nombre de cyclistes approche la dizaine et les coureurs " tournent " pour se relayer (ici dans le sens inverse des aiguilles d'une montre) :

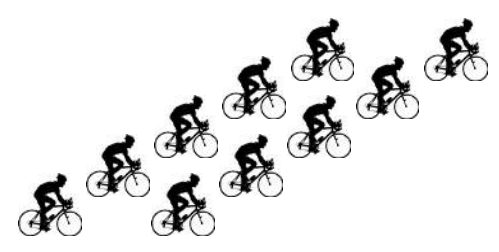

Mais seule une quinzaine de coureurs au maximum peuvent être protégés. Les autres sont dans ce que le jargon appelle "la bordure" :

\section{+}

Les coureurs “bordurés " (en ligne et en bas) ne sont donc pas protégés. Ils roulent dans le fossé pour tenter de trouver un maigre abri. S'ils veulent poursuivre, ils créent alors un deuxième, troisième éventail...

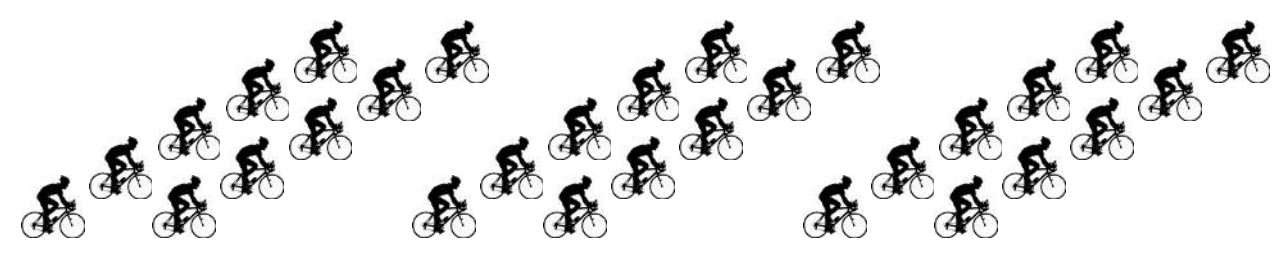

Ainsi quelques mètres se transforment en minutes à l'arrivée, et ce simplement à cause du placement à l'entrée de la zone où " on attrape le vent de côté ". Les grosses équipes savent quand il faut être prêt et organisent ces bordures afin d'être bien représentées à l'avant de la course en "fermant la bordure " (en ne laissant personne d'autre s'abriter). La précision de l'organisation des coureurs de ce niveau dans ces bordures est impressionnante, ils se décalent de quelques degrés après chaque virage pour adapter leurs abris, varient le sens des relais (car la file montante des coureurs qui vont prendre le relais est du côté protégé par la file descendante), ils adaptent leur vitesse, leur temps de relais, ceci en se passant à quelques centimètres pour bénéficier 
du maximum d'abris. Plus le niveau des coureurs est haut, moins il y a besoin de parler, tout au plus un signe du coude suffit-il pour indiquer le passage du relais.

Les pratiquants ont construit des techniques d'organisation afin de s'adapter aux propriétés physiques des épreuves (liées aux lois de l'aérodynamique et de la pesanteur) ainsi qu'aux contraintes biologiques de l'espèce humaine (énergétiques, musculaires, cardio-vasculaires, respiratoires...) tout en respectant les principes de la réglementation sportive et technique.

Le rassemblement de cyclistes les uns derrière les autres découle d'adaptations à des lois physiques et physiologiques. Ces exigences sont pragmatiques puisqu'il s'agit de s'économiser par rapport aux lois physiques de l'aérodynamisme et de l'aspiration d'un corps derrière un autre lorsqu'ils sont en mouvement: "être dans la roue ". Ainsi, des études en mécanique et physique ont calculé qu'à $40 \mathrm{~km} / \mathrm{h}$, un coureur étant dans la roue d'un autre produisait un travail de 30\% inférieur à celui de devant. Au milieu du peloton, l'abri est tel qu'il suffit de donner "quelques coups de pédales" lorsque l'on possède la technique consistant à se "glisser dans les trous". Le "museau" caché dans ces abris, un coureur n'a qu'à " tricoter" pour suivre, même à $50 \mathrm{~km} / \mathrm{h}$. Ce principe adaptatif est également utilisé par les coureurs de rollers de vitesse qui évoluent environ à la même vitesse horaire que les cyclistes et qui ont également un peloton et des codes d'organisation pour réguler les relais. Les cyclistes à VTT ainsi que les skieurs de fond se mettent également dans le sillage de leur concurrent pour bénéficier de l'abri aérodynamique mais ils ne peuvent s'organiser en peloton en raison des caractéristiques des parcours qui exigent de l'adresse et une certaine distance avec les concurrents pour éviter les chutes. Enfin, la tactique des coureurs à pied consiste à ne pas se découvrir trop en évitant de mener un groupe trop tôt, elle peut même mettre un "lièvre " aux avants de la course pour mener un groupe. Mais à la vitesse de la course, soit entre 15 et $20 \mathrm{~km} / \mathrm{h}$, cette adaptation n'est pas aussi nécessaire puisqu'à cette allure, la différence d'énergie dépensée entre le meneur et le suiveur est plus faible que pour les autres activités utilisant des engins favorisant la vitesse et le rendement de la motricité.

Dans le contexte compétitif des courses, la volonté de chaque coureur est bien de gagner la course, si possible en se détachant du peloton. Les compétitions étant longues, chaque coureur essaye au maximum de s'économiser en évitant le plus possible de faire des efforts en étant devant (sauf dans le cas de stratégies spécifiques: course d'équipe, s'échapper pour " montrer le maillot "...). Ainsi, le peloton a établi des principes d'organisation, des valeurs propres à travers sa tradition orale et son jargon. Il s'agit des codes que tout coureur doit connaître pour être accepté. Le respect de ces règles, qui ne figurent pas dans les règlements écrits, conditionne la réputation et l'assignation d'un statut à chaque coureur. 
Ainsi, au sein d'un groupe, les coureurs "se passent des relais" afin que chacun puisse passer le même temps "dans le vent". Pendant ce temps, les autres coureurs peuvent récupérer en étant protégés de la résistance de l'air lié à l'avancement et au vent. La puissance musculaire est réduite, leur consommation énergétique également et logiquement la fréquence cardiaque diminue ce qui permet même la resynthèse des substrats énergétiques et l'élimination de certains déchets métaboliques. A travers ces principes stables, la diversité des situations géographiques en course (vent, relief, état et largeur de la route) engendre des formes typiques du peloton: "éventail-bordure ” lorsque le vent est latéral, par exemple.

Le composé technique qu'est le peloton est donc une tension, une suite de formes créées par ses composants (les coureurs) qui possèdent des intentions concurrentes (chacun veut s'imposer) mais qui s'organisent ensemble. Les formes du peloton ont un sens et révèlent un état de la course. Le peloton est un tout en mouvement avec des files montantes, descendantes; les images météorologiques de masses d'air ressemblent à ces déplacements. Les mouvements et dépressions ne sont pas ici liés à des lois physiques mais au processus correspondant à l'ajustement de la volonté de se placer devant et aux limites physiologiques de chaque coureur. C'est à travers cette co-construction (organisation du composé culturel qu'est le peloton et organisation cognitive et en action de chaque coureur), que ce tout trouve son existence " auto-éco-organisée " (Morin 1977). Mais avec les enjeux, les espoirs de victoire et les rivalités, le peloton n'est pas un groupe homogène, il y règne des tensions et des conflits. Les pratiquants ont donc construit des codes sociaux qui viennent réguler les comportements et surtout hiérarchiser les individus.

\section{LA CONSTRUCTION DE CODES SOCIAUX}

Les caractéristiques physiques de l'effort en cyclisme induisent un rapport à autrui spécifique aux courses sur route. D’une action centrée sur soi caractéristique de certains sports d'endurance, le cycliste engage une action visant à utiliser les autres: "Faut savoir profiter des autres, quand tu vois quelqu'un qui fait un effort pour remonter sur le côté, bah tu te jettes dans la roue." Puisque chaque coureur tente de tirer profit des situations, il existe des règles construites et codifiées par la tradition orale du peloton qui distingue les comportements : utiliser les autres, profiter des autres, usurper une victoire. Ces réputations à connotations hiérarchiques découlent des caractéristiques physiques de l'avancement.

En effet, le fait de profiter d'un adversaire en restant dans son sillage pour s'imposer sournoisement est appelé " ratoner". Avoir un équipier devant soi, un sprinter ou un leader derrière est une excuse valable et autorisée pour ne pas rouler avec un groupe. En revanche, si aucune de ces raisons n'existe, le 
profiteur en question sera traité de " rat", de "raton ", de " ragnole ", des coureurs tenteront de le lâcher, de lui "laisser des trous". Ce comportement est également nommé "sucer la roue " : profiter de l'abri d'un autre, parasiter une échappée comme une sangsue, sans contribuer au travail collectif. Ces comportements déviants conditionnent la réputation d'un cycliste et contribuent à la codification hiérarchique du peloton.

A partir de ce principe, il existe une catégorisation des individus qui s'exprime à travers les termes du jargon. Ils illustrent à travers des métaphores animales et d'autres références des types de comportements et assignent une réputation dans le peloton. La catégorie péjorative des animaux à museau est utilisée pour catégoriser des coureurs qui profitent des autres de façon fourbe. Mis à part le " renard " qui exprime la ruse, les autres sont péjoratifs : le " blaireau " est agressif et belliqueux ; la "fouine" se glisse dans les trous et vole avec fourberie ; le " raton " profite du travail des autres; le " rat " est méprisé, il se dissimule dans les abris et vit au crochet des autres. Les animaux de trait caractérisent des coureurs sympathiques, braves, que le peloton apprécie, dont chacun profite mais se moque ; "le bœuf ", le " bourrin ", roulent devant sans se retourner, sans préoccupation tactique, ils emmènent les plus rusés jusqu'à la victoire, tranquillement installés dans le sillage de ces braves bêtes ; la pauvre " mule " est aussi stupide mais est en plus réputée pour " charger ", c'està-dire utiliser abondamment des adjuvants, souvent sans connaitre les bonnes posologies et les astuces d'un bon remède.

L'organisation sociale est encore plus précise au sein des équipes. Il existe une hiérarchie entre les membres d'une même équipe qui définit les rôles et fonctions de chacun. Cette répartition des tâches est une nouvelle fois adaptée à la caractéristique de l'effort. Ainsi, tout va être réalisé pour que les plus forts, les spécialistes (grimpeur, puncheur ou routier-sprinter) de l'épreuve du jour soient protégés par les autres, qu'ils s'économisent au maximum pour la victoire. Les “porteurs de bidons" les ravitaillent pour leur éviter les efforts qu'occasionnent la remontée du peloton, les "locomotives, machines à rouler" sont disponibles pour " tirer des grands bouts droits ", c'est-à-dire rouler en tête de peloton pour revenir sur une échappée, au détriment de leur réussite personnelle. Ces qualités sont appréciées pour être recruté comme bon "équipier ", soit comme domestiques. Ces derniers qualificatifs correspondent au statut des sans grade, au potentiel banal dans le peloton. Ce terme est à la fois rabaissant, utilisé avec ironie et à la fois valorisant car il souligne le professionnalisme, l'abnégation et l'appartenance à l'équipe. Cette organisation sociale, qui associe le dévouement des équipiers et les résultats des leaders, se régule par des dons d'argent aux équipiers mais également par la confiance et la reconnaissance au sein du groupe. Un équipier trouve un prestige dans son rôle: "il est l'équipier de ", "il est dans l'équipe ", "il est professionnel ou élite ". Il faut également noter que le statut de leader peut évoluer au fil 
de la saison en fonction des spécialités de chacun. Un grand routier-sprinter peut se mettre au service d'un grimpeur sur une course de montagne et réciproquement, ce même grimpeur peut rouler pour son coéquipier afin de préparer un sprint sur une course plus plate, d'où l'adage " le cyclisme est un sport individuel qui se pratique en équipe ". Ce dévouement et ces pratiques de don de soi consolident le groupe, créent du lien social et permettent également d'attendre une aide des autres membres de l'équipe en retour. Ainsi, on constate que le cyclisme d'élite nécessite de s'adapter au collectif, de présenter des compétences sociales au sein de l'équipe et pas seulement des compétences physiques - l'article du sociologue Williams (1989) au sein du cyclisme canadien converge également dans ce sens.

\section{DES ORGANISATIONS SOCIALES PARTICULIÈRES ET COUTUMIÈRES}

Le premier extrait ethnographique évoque le "grupetto ", un groupe où les battus, les exclus de la performance créent un réseau de solidarité où les codes et les valeurs établies sont en opposition radicale avec la course. Le train est assuré pour que le maximum de coureurs arrivent dans les délais, puissent repartir pour l'étape du lendemain et ne pas quitter son équipe, ses fonctions. Il s'agit d'un temps de transition où l'espoir de jours meilleurs entretient la bonne humeur et facilite les contacts. Les concurrents lient souvent des affinités dans ces moments de défaillance et de solidarité. Certains, réputés sympathiques et généreux dans l'effort pour être la locomotive de cet "autobus", sont parfois même favorisés ultérieurement quand ils s'échappent sur des courses de plaine. Le peloton peut avoir un traitement de faveur en laissant un "bon de sortie" et en observant un temps de latence avant d'entamer une poursuite effrénée, commandée par les directeurs sportifs.

Le deuxième extrait décrit la mise en place d'une "mafia ". Ce type d'organisations illicites a principalement lieu sur les critériums estivaux. Notons tout de même que cette conception du cyclisme ne fait pas consensus. L'extrait montre les tensions entre les concurrents qui s'opposent, entrent en conflits de valeurs. Mais, ces formes d'organisations illicites et coutumières fonctionnent souvent par réseaux de coureurs expérimentés qui vivent de la pratique, même en étant amateur - c'était d'ailleurs le cas du chef de cette " mafia".

Il existe également ce type de procédé dans les courses par étapes lorsqu'un coureur a le maillot de leader du classement général. Il arrive parfois qu'au fil des étapes, son équipe soit décimée, réduite ou affaiblie. Ce coureur peut aller voir des connaissances d'une équipe concurrente qui n'a plus rien à espérer de la course (placés aux 30 ${ }^{\text {ème }}, 60^{\text {ème }}$ places...). Il propose de les " embaucher ", en leur donnant, par exemple, 100 ou 1000 euros selon le niveau de l'épreuve afin qu'ils roulent pour lui sur les échappées, et qu'ils impriment " un tempo " afin de neutraliser la course. Les commissaires n'ont aucun moyen de contrôle. 
Il n'est pas interdit à un coureur de rouler devant le peloton sans raison stratégique... Les connaisseurs remarquent ce qui se passe mais il est impossible d'intervenir ! Lors des épreuves télévisées, les consultants experts connaissent bien évidemment ce phénomène mais tentent de conserver le secret: "C'est peut-être le directeur sportif qui leur a demandé de préparer le contre-la-montre par équipe de demain ? ". Il est également fréquent qu'un leader du classement général donne une victoire d'étape à un adversaire pour mieux le contrôler, amadouer la rivalité. Lorsqu'un coureur bien placé au général s'échappe en compagnie d'un autre coureur qui est mal classé, il lui offre la victoire d'étape en ne réalisant pas le sprint final en contre-partie de ses efforts pour augmenter les écarts de temps avec le peloton à l'arrivée.

Le respect de ces valeurs s'effectue par la crainte des représailles et de la réputation assignée à ceux qui ne respectent pas ces codes. Ainsi, la domination de certains coureurs sur d'autres n'est pas sans évoquer le rapport d'un suzerain avec ses vassaux à qui il peut accorder une protection, voire une petite victoire dans l'attente d'un soutien lors d'un grand et prestigieux affrontement.

Le schéma organisationnel du peloton oscille entre une forme officielle et officieuse. D’un côté des structures nationales et internationales protégées par les institutions de contrôle réglementaire, de l'autre une régulation spontanée, populaire, parfois illégitime. Le sport cycliste institutionnalisé et réglementé selon les valeurs de l'éthique sportive débouche sur une construction culturelle coutumière, ayant élaboré une tradition orale ainsi que des procédés de coercition et parfois de corruption.

\section{DES VALEURS, UNE ORGANISATION ET UNE CULTURE TECHNIQUE ATYPIQUE}

L'exigence physique des épreuves a également induit une adaptation technique des pratiquants pour supporter la difficulté, la longueur et la répétition des épreuves. Un coureur professionnel réalise plus de 35000 kilomètres par an. La tradition du peloton transmet donc des "techniques du corps" (Mauss 1968) pour tous les domaines de la vie quotidienne.

Au delà des techniques d'entraînement, les soins du corps font l'objet d'une organisation avec les onctions d'avant-course d'huiles à base de camphre et les massages le soir, à l'hôtel. Ces soins corporels se caractérisent par le rasage des jambes. Il possédait à la base une fonctionnalité, celle de faciliter la cicatrisation en cas de chute mais il est rapidement devenu un marqueur de l'esthétique corporelle du coureur qui se doit également de présenter une musculature "affûtée ", sans apparence de gras et avec des muscles " secs". Cela exige donc une alimentation surveillée à base de féculents comme les pâtes et de nombreux compléments alimentaires (protéines, vitamines, minéraux) sous formes de cachets. 
Ces techniques de consommation associent pour certains, selon le niveau et l'encadrement, quelques adjuvants. Cette consommation, allant du " bricolage " occasionnel à l'utilisation suivie médicalement, s'insère dans des organisations coutumières au sein des équipes ou par la formation de groupuscules organisés pour l'approvisionnement de produits dopants. Cette organisation coutumière est traditionnelle dans le peloton. Dès les premières compétitions de la fin du $19^{\text {ème }}$ siècle, des soigneurs et personnages préconisaient des remèdes que les coureurs s'échangeaient. Les frères Pélissier en parlent publiquement dans le célèbre article d'Albert Londres, “Les forçats de la route ”, dès 1924 :

Voulez-vous voir comment nous marchons ? Tenez...

De son sac, il sort une fiole :

- Ça, c'est de la cocaïne pour les yeux, ça, c'est du chloroforme pour les gencives...

- Ça, dit Ville, vidant aussi sa musette, c'est de la pommade pour me chauffer les genoux.

- Et des pilules? Voulez-vous voir des pilules? Tenez, voilà des pilules.

Ils en sortent trois boîtes chacun.

- Bref, dit Francis, nous marchons à la dynamite (Londres 1980 [1924]: 3).

Le phénomène du dopage a pris une autre dimension depuis les années 1990. La rencontre entre le sport de haut niveau avec le contexte économique et les bio-technologies a conduit à un scénario où les champions sont également des prototypes pour certains chercheurs en génie génétique. Mais ce témoignage des frères Pélissier mentionne déjà la prise de cocaïne. Les témoignages de coureurs amateurs actuels transmettent cette tradition à travers les nombreux termes du jargon : " taper dans le pot ", " user de la fléchette ", " gober ", " saler la soupe ". Le deuxième extrait mentionne également un témoignage de cette prise occasionnelle d'adjuvants à travers cette tradition coutumière : " il roule pas avec nous et il nous fait les primes, heureusement que j'étais pas chargé aujourd'hui sinon je l'aurais mis dans le fossé ".

Là encore, il existe des conflits de valeurs, puisque certains coureurs refusent la prise de produits. Cette tradition technique est notamment en évolution en raison des nombreuses affaires de dopages.

Au delà des conflits entre protagonistes du cyclisme, qui manifestent le dynamisme et les interactions des valeurs du peloton avec les évolutions de la société, il se dégage indéniablement une culture du peloton à travers un idéal commun, une esthétique corporelle, une culture technique, une tradition orale, une organisation sociale avec ses propres codes hiérarchiques. 


\section{UN BOUILLON DE CULTURE EN INTERACTION AVEC LES INSTITUTIONS ET LES REPRÉSENTATIONS SOCIALES}

Il apparait donc que le peloton a structuré une culture possédant son organisation sociale, ses codes hiérarchiques et ses valeurs à partir du rapport au corps et à l'acte spécifique du cyclisme sur route. Les caractéristiques physiques de cette activité ont induit des adaptations techniques et des codes sociaux particuliers.

Les propriétés physiques, techniques et réglementaires, également nommées "propriétés formelles" (Darbon 2002) et "propriétés physiques et intellectuelles" (Bromberger 1995b) sont donc à la fois structurées par un système social, technologique et structurantes d'une forme culturelle nouvelle. Si une pratique ne se résume pas aux modalités historiques qui ont contribué à sa construction, en revanche, les propriétés réglementaires et techniques qui en découlent sont à l'origine de l'élaboration des traits de la culture sportive des pratiquants. Pour autant, il n'y a pas une symétrie entre la construction historique fondatrice des propriétés et la culture sportive qui en découle. Il y a au contraire une imprévisibilité des constructions culturelles des pratiquants qui s'adaptent et créent des formes techniques et sociales inédites.

La présentation d'éléments ethnographiques du peloton cycliste illustre qu'il y a non seulement des constructions culturelles nouvelles, mais également l'élaboration de valeurs et de pratiques sociales qui viennent en opposition avec certaines des valeurs fondatrices réglementaires. Cet équilibre instable entre la culture des pratiquants et les normes des instances fédérales garantes des principes officiels se manifeste par des tensions et des conflits. Le sport cycliste en témoigne actuellement avec de nouvelles réglementations en matière de lutte anti-dopage qui engendrent, pour certains coureurs, un changement de pratique et un renoncement de la prise d'adjuvants, pour d'autres, des efforts accrus dans l'utilisation de produits pharmaceutiques encore inconnus des laboratoires de recherche des instances institutionnelles. Il y a donc des évolutions de règlements pour répondre aux nouvelles pratiques des acteurs de terrain et conserver le contrôle de l'activité. Certaines évolutions réglementaires proviennent également de la volonté des institutions sportives d'adapter la pratique sportive au contexte du public, de la société. Ces nouvelles réglementations induisent ensuite des adaptations des pratiquants... Les propriétés d'un sport constituent donc le noyau dynamique et évolutif d'interactions socio-institutionnelles. Elles permettent, soit, de remonter vers les valeurs et les représentations de la société, soit, de plonger vers l'étude de la culture sportive des pratiquants. 


\section{BIBLIOGRAPHIE}

BROMBERGER, C., 1995a, Le match de football: ethnologie d'une passion partisane à Marseille, Naples, Turin. Paris, Editions de la Maison des Sciences de l'Homme.

— 1995b, “De quoi parlent les sports ”, Terrain, 25: 5-12.

DARBON, S., 2002, "Pour une anthropologie des pratiques sportives : propriétés formelles et rapport au corps dans le rugby à XV", Techniques et culture (Sports et corps en jeu), 39: 8-31.

GARNOTEL, X., 2009, Le peloton cycliste: ethnologie d'une culture sportive. Paris, L'Harmattan.

LAGRUE, P., 2004, Le Tour de France : reflet de l'histoire et de la société. Paris, L'Harmattan.

LONDRES, A., 1980 [1924], “Les forçats de la route ”, Recherches [Aimez vous les stades? Les origines historiques des politiques sportives en France (1870-1930), ed. A. Ehrenberg], 43: 3-6.

MAUSS, M., 1968, “Les techniques du corps ”, Sociologie et anthropologie. Paris, PUF, 365$-386$.

MORIN, E., 1977, La méthode, 1. La nature de la nature. Paris, Editions du Seuil.

SAOUTER, A., 1995, "La maman et la putain : les hommes, les femmes et le rugby", Terrain, 25: 13-24.

SEGALEN, M., 1994, Les enfants d'Achille et de Nike : une ethnologie de la course à pied ordinaire. Paris, Métaillé.

WACQUANT, L., 2002, Corps et âmes : carnets ethnographiques d'un apprenti boxeur. Marseille, Agone.

WILLIAMS, T., 1989, "Sport, hegemony and subcultural reproduction : the process of accommodation in bicycle road racing ", International Review for the Sociology of Sport, 24: 315-331.

The bunch of cyclists: from social metaphor to culture medium - Xavier Garnotel - Institut d'Ethnologie Méditerranéenne Européenne et Comparative, Aix-en-Provence • xavier.garnotel@ orange.fr

This article presents at first the integration of cycling as a sport in the technical and social structures of Western societies. Ethnographical notes describe then the life of the cycling bunch and the practices of the riders. These descriptions illustrate an atypical culture which sometimes does not correspond to the statutory texts. The analysis is then focused on the riders' adjustment to the characteristics of the practice and to the construction of social codes and a specific technical culture.

KEYWORDS: ethnology, culture, sport, bunch, cycling, regulation. 